\title{
Significance of Simultaneous Use of Different Methods for Interpretation of Internal Quality Control Data: An Experience of a Tertiary Care Hospital
}

\author{
Shakti Kumar Yadav', Rupinder Kalra ${ }^{1}$, Aarzoo Jahan ${ }^{1}$, Sonam Kumar Pruthi', \\ Namrata Sarin ${ }^{1}$, Sompal Singh ${ }^{1}$ and Harsh Vardhan Singh ${ }^{2 *}$
}

\begin{abstract}
${ }^{1}$ Department of Pathology, North Delhi Municipal Corporation Medical College and Hindu Rao Hospital, New Delhi India ${ }^{2}$ Department of Biochemistry, North Delhi Municipal Corporation Medical College and Hindu Rao Hospital, New Delhi India
\end{abstract}

\section{ABSTRACT}

Background: Quality control is an essential part of quality management in medical laboratory.Various methods are available for the analysis of laboratory quality control data, common ones are Levey Jennings chart and Westgard rules. Westgard multi-rules are a set of rules based on combination of criteria to decide whether an analytical run is acceptable or unacceptable. CUSUM uses the cumulative sum of deviations from a target, however, it is rarely used in current medical laboratory practice. We share our experience of using Westgard rules and CUSUM in the analysis of laboratory quality control data.

Material and Methods: Internal quality control values of 2-year period was included in the present study. Data for platelet count values of normal level control material run on a fully automated haematology analyser(Sysmex XT-2000i) were analysed. A total of 1825 data points were obtained. The data was interpreted by Westgard rules as well as cumulative sum method. The out of control events was analysed.

Results: There was 9 incidence of control value outside 3SD (Westgard Rule 13s) which was picked on Levey Jennings chart but missed by CUSUM method. There were 22 instances of shift in mean (bias) which were only picked by CUSUM method.

Conclusion: CUSUM was more sensitive for detection of bias whereas random error was picked-up early by Westgard rules. In conclusion we recommend the use of more than one method for analysis of quality control data.

\section{Keywords: Quality Control, LJ Chart, CUSUM, Westgard Rules}

\section{Introduction}

Quality control is an essential part of quality management in medical laboratory. The guideline for the same has been laid down in Guidelines for Good Clinical Laboratory Practices (GCLP), Indian Council of Medical Research, New Delhi, India. ${ }^{[1]}$ The guideline prescribes the use of Westgard multi-rules for the interpretation of the daily control values. These rules help decide whether to accept or reject the test run. ${ }^{[2]}$

Majority of the medical laboratories rely on the quality control samples for Internal quality control to ensure accuracy and precision of the laboratory reports. The QC protocol is based on the schedule of number of times and number of levels the internal quality control run. The IQC guidelines are usually provided by the national and international accreditation bodies.

Even though the task is to keep the measurement error at the minimum, it can never be truly nullified and quality control methods are used to decrease errors to an acceptable limit. ${ }^{[3]}$ Each type of quality control method is also prone to errors- both random and systematic error.
Random errors are unpredictable. They may occur due to issue in instruments, sample or analytical process. ${ }^{[4]}$ Systematic error also known as bias is consistently affects the result and indicates a problem in the analytical process. Both types of errors have a cumulative effect on the final result. ${ }^{[3]}$

Various methods are available for the analysis of laboratory quality control data, common ones are Levey Jennings chart (LJ chart), Westgard rules. ${ }^{[5]}$

In $\mathrm{LJ}$ chart, the daily control values are plotted on y-axis while time is plotted on $\mathrm{x}$-axis. The mean and one, two and three standard deviation limits are also plotted on y-axis. Thus, LJ chart provides a simplistic way to monitor the changes in control values. ${ }^{[6]}$

Westgard multi-rules are a set of rules based on combination of criteria to decide whether an analytical run is acceptable or unacceptable. ${ }^{[7]}$ CUSUM uses the cumulative sum of deviations from a target. The CUSUM chart plots the cumulative sum of deviations from the target for individual measurements. It is used to monitor small shift in process mean, however it is less used. ${ }^{[8]}$ We share our experience 
of using Westgard rules and CUSUM in the analysis of laboratory quality control data.

The aim of present study was to compare the CUSUM chart and LJ Chart with Westgard rules for the detection of errors in medical laboratory quality control.

\section{Material and Methods}

The study was conducted in a tertiary care hospital having 1000 beds. The haematology section of the hospital receives samples from both out-patients and in-patient departments. The section is currently having five 3-part automated haematology analyser (four Sysmex KX-21 Cell Counter, Sysmex America Inc. Illinois USA and one Celltac $\alpha$, Nihon Kohden, Japan). Both qualitative (opinion on microscopic examination of peripheral blood smear) and quantitative (complete blood count) reports are generated.

The laboratory has in place both quality management systems for external and internal quality control. For external quality assurance, our hospital has subscribed to the hematology EQAs system of premier medical college hospital of our country. Internal quality control is done using conventional control material supplied by the manufacturer (Eightcheck-3WP, Sysmex Europe GmbH).

Control sample is run for each of the three batches daily and LJ chart are plotted and interpreted using Westgard Rules for internal quality control. Although, CUSUM plot was made, it was not used for any corrective action, since, we did not have any prior experience of application of CUSUM for quality control.

For CUSUM control chart application we cumulated the upper and lower standardized deviations as shown in equation 1 . The standardized deviations were modified by a factor $\mathrm{k}=0.5$, and upper and lower cumulative sums were calculated as shown in equation 2 and 3.

Following method was used to plot CUSUM chart.

1. Standardized deviation $\mathrm{z}_{\mathrm{i}}$ was calculated using formula

$\boldsymbol{z}_{\boldsymbol{i}}=\frac{\boldsymbol{x}_{\boldsymbol{i}}-\overline{\boldsymbol{x}}}{\boldsymbol{S D}} \quad$ [Equation 1]

Where, is the value of control material obtained in a particular run, is laboratory mean and SD is standard deviation of the control material.

2. Upper cumulative sum $\left(\mathrm{S}_{\mathrm{H}}\right)$ and lower cumulative sum $\left(\mathrm{S}_{\mathrm{L}}\right)$ was calculated as

$S_{H}=\max \left[0,\left(z_{i}-k\right)+S_{H-1}\right] \quad$ [Equation 2]

$S_{L}=-\max \left[0,\left(-z_{i}-k\right)+S_{L-1}\right] \quad$ [Equation 3]
Where,

$\mathrm{S}_{\mathrm{H}-1}$ was upper cumulative of previous value

$\mathrm{S}_{\mathrm{L}-1}$ was lower cumulative of previous value

Upper and lower reference limits in the present study were set as +6 and -6 respectively.

For situations where $\mathrm{S}_{\mathrm{H}}<0$ and $\mathrm{S}_{\mathrm{L}}>1$, their values were reset to 0 .

For present study we acquired control sample data from normal level control for platelet count over a period of two years. Before the start of every new batch of control material the laboratory mean and standard deviation (SD) for the assessed parameter were estimated by running it several times.

\section{Results}

A total of 1825 data points were analysed. The monthly coefficient of variance $(\mathrm{CV})$ for the assessed parameter ranged from 5.6 to $7.3 \%$ during the study period._On analysing the data, we found that out of 1825 data points a total of 31 events showed error.

Out of these, nine event violated rule $1_{3} \mathrm{~s}$ (random error) and 22 were in violation of rule 10x (bias). Root cause analysis (CAPA analysis) was done for every error event. Out of nine random events six were due to human error in handling of the control sample and three were due to machine failure (e.g. from tubing, failure of electronic circuit and software related issues).

Out of 22 bias events, 18 were due to changes in control material especially towards the end of shelf-life and five events were due to machine error.

All of the nine random errors were picked on Levey Jennings chart but missed by CUSUM method. There were 22 instances of shift in mean (bias) which were only picked by CUSUM method (Figure 1). For all instances of machine error engineer was called for repair and maintenance after which inter machine comparison was done to validate the equipment functioning. Since the CUSUM method was used for the first time in our laboratory, action was only taken based on Westgard rules and no intervention was done for error on CUSUM report.

\section{Discussion}

The field of laboratory medicine has evolved in the era of evidence-based medicine. It requires optimal data from the laboratory processing results which can be significant for the patient health and treatment. Therefore, monitoring of the quality control practices and methods is always required to avoid suboptimal performance. 


\section{CUSUM Plot Test Name: Platelet (Test Code: 219)}

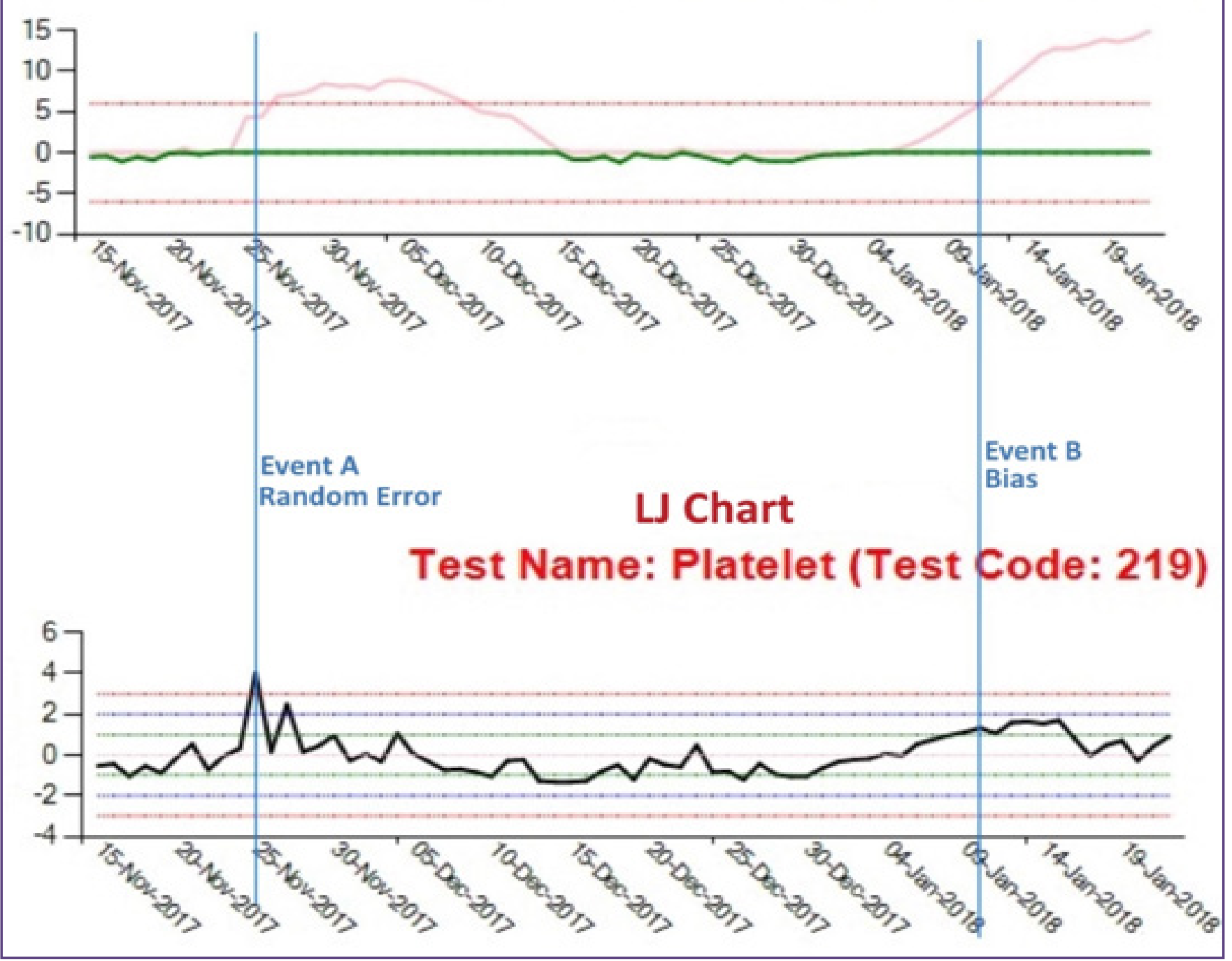

Fig. 1: LJ chart and CUSUM Plot depicting that random error (event A) was picked on LJ chart using Westgard rule 13s but it was missed on CUSUM plot. The bias (event B) was under the 12s rule and its was not picked by Westgard rule but it was picked on the CUSUM plot.

As quality control has now become an essential part of quality management in medical laboratories around the world. It becomes crucial that such methods fulfil certain criteria for optimal performance.

Various methods for the analysis of quality control data are available for use. Tools which are in common use for laboratory internal quality control include Levey-Jennings charts (Shewhart Charts), Westgard rules, Average of normal etc. All these tools compare the performance of an analytical method in order to achieve relevant quality goals.
Walter Shewhart in 1924 developed two types of control charts, one based on mean value of samples [ $\overline{\mathrm{x}}$ - Shewhart chart/Average Shewhart chart $\}$ and second was range of sample values [R-shewhart chart]. These charts were intended to be used for process control in Production and Manufacturing sectors.

CUSUM method was first described by Page et al. ${ }^{[9]}$ for both surveillance and quality control. CUSUM is one of simple statistical methods. It provides rapid analysis of data and helps in identification of trends in data set or 
series of data. CUSUM plot can be utilised for any data which is gathered serially. Haely in 1968 advocated the use of CUSUM in medical laboratory quality control. ${ }^{[8]}$ Still CUSUM charts are seldom used in medical Laboratories. This lack of acceptance of CUSUM was due to need to maintain these charts and also because of the qualitative manner of interpretation. ${ }^{[5]}$ Availability of computerized systems in modern medical Laboratories implementation of CUSUM chat has now become simpler. Hence, CUSUM method might prove to be easier method which can be applied for quality control.

In 1950, Stanley Levey and Elmer Jenning adapted average schewart chart for chemical analysis in medical laboratory.

${ }^{[5]}$ This new chart presently known as LJ chart has become a primary tool for internal quality control across medical laboratories. In 1917, James Westgard et. al. developed variations of CUSUM chart called as decision limit CUSUM chart for better adaptability as per requirement of clinical chemistry. ${ }^{[7]}$ Later in 1981, Westgard published multi rule schewart charts for quality control in medical laboratory. This established simple rules by which medical Laboratories able to quickly interpret the LJ charts. These multi-rules you were intended to distinguish between random errors and systemic errors.

However, Westgard rule may not be able to detect some errors as there is always a threshold point is needed to be crossed before an event is triggered after which intervention is done.

In present study, the CUSUM chart was able to detect errors which were under the $2_{\mathrm{S}}$ Westgard rule. Westgard et al. also showed similar findings with CUSUM chart being more sensitive for the detection of systematic shift and drifts. ${ }^{[7]}$ He also mentioned that the probability of error detection can be doubled with the use of combined Shewhart Chart. He proposed a decision limit CUSUM method for use in medical and chemical laboratories. ${ }^{[10]}$

Presently, the CUSUM charts are used to analyse outcomes in a variety of clinical applications. Fortea-Sanchis et al. applied CUSUM control chart to assess the quality control techniques in surgical procedure and concluded that CUSUM chart can be utilized to obtain cut-off points for quantitative variables and to monitor care activity. [11] Gould AL signified the role of CUSUM chart in the monitoring of adverse event count frequencies from single and blinded trials. CUSUM charts can be used to monitor such events with out unblinding the ongoing trials. However conventional statistical method cannot be applied on blinded trials. ${ }^{[12]}$ Righi L et al. used CUSUM controls charts for monitoring of antimicrobial resistance and found its use to be complimentary for the hospital infection control strategies. ${ }^{[13]}$ Rasmussen $\mathrm{TB}$ et al applied risk adjusted CUSUM charts for the monitoring of the 30 day hospital mortality and found that a alarm from CUSUM chart can used identify performance problem. ${ }^{[14]}$

Sampson ML used CUSUM with logistic regression (CSLR) method to predict the testing errors and found that it to a rapid and sensitive detection method for laboratory errors. ${ }^{[15]}$

Nightingale, M. J compared the use of conventional Shewhart chart and CUSUM chart in quality monitoring of blood components and found that CUSUM was more sensitive in detecting small changes as compared to Shewhart charts. ${ }^{[16]}$ These findings are in concordance with the findings of the present study.

Woodhall et al. pointed out that a basic Shewhart control chart like LJ chart can have a possibility of false alarm at each sampling point. However, charts which accumulates information over time e.g. CUSUM chart are not easily affected by metrics of false alarm. ${ }^{[17]}$

\section{Conclusion}

In present study we found that, as compared to Westgard rules CUSUM is more sensitive for detection of systematic error (bias). However, random errors have a greater chance to be picked-up early by Westgard rules. In conclusion, more than one method for analysis of quality control data must be used. This will help in keeping both systematic as well as random errors in control, and will assure optimal quality control in medical laboratories.

\section{Acknowledgement}

Nil

\section{Funding}

Nil

\section{Competing Interests}

None declared

\section{References}

1. Selvakumar R. Good laboratory practices. Springer; 2010.

2. Guidelines for good clinical laboratory practices (GCLP). New Delhi: Indian Council of Medical Research; 2008.

3. Momeni-Boroujeni A, Pincus MR. Systematic Error Detection in Laboratory Medicine. Quality Control in Laboratory. 2018:49.

4. Momeni A, Pincus M, Libien J. Introduction to statistical methods in pathology: Springer; 2018.

5. Karkalousos P, Evangelopoulos A. The history of statistical quality control in clinical chemistry and haematology (1950-2010). Int J Bio Lab Sci. 2015;4:1-11. 
6. Levey S, Jennings ER. The use of control charts in the clinical laboratory. Am J Clin Pathol. 1950;20:1059-66.

7. Westgard JO, Barry PL, Hunt MR, Groth T. A multi-rule Shewhart chart for quality control in clinical chemistry. Clin Chem. 1981;27:493-501.

8. Chang WR, McLean IP. CUSUM: a tool for early feedback about performance? BMC medical research methodology. 2006;6:8.

9. Page E. Cumulative Sum Control Charts. Technometrics 1961;3:1-9.

10. Westgard J, Groth T, Aronsson T, De Verdier C. Combined Shewhart-cusum control chart for improved quality control in clinical chemistry. Clinical Chemistry. 1977;23:1881-7.

11. Fortea-Sanchis C, Escrig-Sos J. Quality Control Techniques in Surgery: Application of Cumulative Sum (CUSUM) Charts. Cirugía Española (English Edition). 2019;97:65-70.

12. Gould AL. Control charts for monitoring accumulating adverse event count frequencies from single and multiple blinded trials. Stat Med. 2016;35:5561-78.
13. Righi L, Amarsy R, Picat MQ, Thuillier M, Cambau E, Raskine L, et al. Monitoring antimicrobial resistance (AMR) using CUSUM control charts. European Journal of Clinical Microbiology \& Infectious Diseases. 2017;36:1519-25

14. Rasmussen TB, Ulrichsen SP, Nørgaard M. Use of riskadjusted CUSUM charts to monitor 30-day mortality in Danish hospitals. Clin Epidemiol. 2018;10:445-56.

15. Sampson ML, Gounden V, van Deventer HE, Remaley AT. CUSUM-Logistic Regression analysis for the rapid detection of errors in clinical laboratory test results. Clin Biochem. 2016;49:201-7.

16. Nightingale MJ, Madden S, Curnow E, Collett D, Procter $\mathrm{S}$, Rowe GP. An evaluation of statistical process control techniques applied to blood component quality monitoring with particular reference to CUSUM. Transfus Med. 2012;22:285-93.

17. Woodall WH, Faltin FW. Rethinking control chart design and evaluation. Quality Engineering. 2019;31:596-605.

*Corresponding author:

Dr. Harsh Vardhan Singh, Department of Biochemistry. North Delhi Municipal Corporation Medical College and Hindu Rao Hospital, New Delhi India Email: hvsingh77@gmail.com

Financial or other Competing Interests: None. 